\title{
Teaching Method Research on the Parameters of Adjustment and Control for "Building Structure Computerization"
}

\author{
Feng Zhengwei \\ Department of Civil Engineering \\ Xiamen University Tan Kah Kee College \\ Fujian, Zhangzhou, PR of China, 363105 \\ Zhang Rongjie \\ Department of Civil Engineering \\ Xiamen University Tan Kah Kee College \\ Fujian, Zhangzhou, PR of China, 363105
}

\author{
Lin Longbin* \\ Department of Civil Engineering \\ Xiamen University Tan Kah Kee College \\ Fujian, Zhangzhou, PR of China, 363105 \\ *llb@xujc.com \\ Wu Tianming \\ Department of Civil Engineering \\ Xiamen University Tan Kah Kee College \\ Fujian, Zhangzhou, PR of China, 363105
}

\begin{abstract}
There are many problems in the teaching of "Building Structure Computerization", such as students' difficulty in comprehension and mastering, too many design parameters and so on. For these problems, we summarize and classify all kinds of the calculation parameters. The principle, value and influence on the calculation results about the number of modes and the period shortening factor are introduced. The connotation and significance of the period ratio, the displacement ratio and the axis compression ratio in the output text of Space Analysis of Tall-Buildings with Wall-Element (SATWE) postprocessing are analyzed, and the method of control and adjustment are proposed. Through this improvement of teaching, the difficulty of learning courses is reduced, the confidence and interest of the students learning are enhanced, and the principle and application of the design parameters can be mastered by students easily. The class efficiency have been greatly improved, this teaching methods play a very good effect.
\end{abstract}

Keywords-Mode number; Period shortening factor; Period ratio; Displacement ratio; Axial compression ratio

\section{INTRODUCTION}

At present, the graduation design of civil engineering major in most colleges is modeled and calculated by Bent Frame and Plane Aided Design (PKPM) software, and offer the computer courses about "Building Structure Computerization". The purpose will reflect the combination of teaching and practice better, and cultivate the ability of students to solve practical problems with professional knowledge, so that students can adapt to their jobs fast after graduation.

PKPM software can be divided into pre-processing module (model building), analysis module (design parameters and the definition of special components), post-processing module (graphics and text files) and the construction drawing. In the process of structural analysis and calculation, it is necessary to select the parameters of PKPM reasonably, combine with the actual project and the use of procedures to obtain the

This work was supported by the education science "13th Five-year Plan" key topic of Fujian Province of China (No. FJJKCGZ16-069). reasonable results. If the design parameters are inaccurate or wrong, it will affect the accuracy, reliability and practicability of the calculation results, and affect the safety of the designed buildings in severe cases [1].

In the course of teaching, the calculation parameters are divided into two categories: one is the input design parameters of Space Analysis of Tall-Buildings with Wall-Element (SATWE) before the model is calculated; the other is the control parameters in the calculation results. According to the standard of high-rise building, the basic meanings and values of the important parameters are introduced, the influence of the calculation results about several important parameters of SATWE are analyzed, and put forward the control and adjustment method about SATWE post-processing parameters output text.

\section{PROBlEMS IN THE TEACHING PROCESS}

The teaching of "Building Structure Computerization" mainly composes of two parts, modeling and structural calculation. The modeling part is simple and easy to learn because of strong operability, the students' enthusiasm for learning is very high. The structure calculation involve the input control of the parameters and the adjustment of the parameters after the end of the calculation, which should combine with the standard provisions and professional knowledge, large amount of information, it is difficult for students to master.

For the first kind of parameters, the most students will refer to the specification, but don't understand the real meaning and the influence on the results for some complex parameters. The students only copy blindly to imitate the input data about the second kinds of parameters, don't know how to adjust the unsatisfactory results, the ability of handling the late problem is poor. 


\section{Setting of SATWE Design Parameters}

Before starting the calculation, the initial parameters should be correctly selected and defined according to the specification and the project practice. SATWE pre-processing includes many items, and there are many parameters in "Supplementary definition of analysis and design".

Because of the large amount of information, the students are not easy to master in learning. Combined with the experience of curriculum teaching, the classification of parameters is more conducive to students learning. In this paper, according to the nature of use the calculation parameters of structure and component are divided into the following four categories: (1) ease of use the parameters that can be found by the building code, such as material bulk density, wind load information, live load reduction factor, seismic grouping, seismic intensity and damping ratio and other related parameters, (2) the actual project information has been given, such as the basement layer numbers, structural system, the weak layer settings etc, (3) the parameters are needed to calculate multiple times, It is difficult to estimate before calculation, which need to be calculated by trial, such as the direction of the maximum earthquake force, mode number, the basic cycle of the structure etc, and (4) the parameters need to be considered selectively, such as rigid floor assumption, period shortening factor, occasional eccentricity, bi-directional earthquake motions, the moment redistribution ratio at beamend, the rigidity amplification coefficient of center beam etc.

It is easy to find and master the parameters by students that can be read by the specification and the actual project. Some parameters are related to the results of the whole calculation, so it is necessary to take reasonable values to ensure the correctness of the calculation results. A part of students can not use the parameters well because of the unclear concept, such as mode number, period shortening factor and occasional eccentricity etc.

\section{A. Mode Number}

The numbers of mode are directly taken 3 times the floor, when most students set the parameters. But it is not clear that the meaning of the parameters and the influence of the parameters on the calculation results.

In the calculation of seismic force, the number of mode shapes should be the number of modes required to participate in the mass to reach $90 \%$ of the total mass, and related to the number of layers and the structure. It takes 3 times layer, multistorey building is larger than or equal to 3 , high-rise buildings is at least 9. When the torsion coupling calculation is considered, the mode number are less than or equal to 3 times the total layer, the mode number of multi-tower structure are more than 9 times the number of towers [2]. There are at most 3 effective dynamic degrees of freedom on one floor, so there are at most 3 modes. The number of modes can not exceed the total number of vibration modes of the structure, it will cause abnormal seismic force calculation if the modes exceed.

With the increase of the mode number, the effect of horizontal seismic action increases. The number of mode shapes are less, the seismic effect caused by the subsequent mode shapes are not included, which lead to the calculation result is not safe. The number of mode are more, which lead to the long operation time and high memory requirements for the computer, the extra high vibration mode have little contribution to the structural seismic action [3].

In many high-rise buildings, the mode number can be 3 times the layer. When the students set the parameters, the calculation results should be look for the effective mass coefficient is greater than $90 \%$, otherwise the number of modes should increase to be recalculated. The mode number of multitower structure are more than 9 times the number of towers, it check the effective mass coefficient at the same time.

\section{B. Period Shortening Factor}

Most students choose the period shortening factor according to the standard data, but ignore the material composition of the infill wall. The reduction coefficient is given in "Technical Specification for Concrete Structures of Tall Building", but mainly for solid clay brick wall. It doesn't give the widely used aerated concrete infill wall, ordinary concrete and lightweight aggregate concrete hollow block filling wall, clay hollow block filling wall and so on. It will lead to a certain gap between the calculation results and the actual project if the parameters are selected according to the standard.

The period shortening factor is inversely proportional to the stiffness of the infill wall, the overall stiffness is greater, the period shortening factor is smaller. In the process of parameter setting, the period shortening factor is too large, so that the seismic behavior of the structure is too small, the design is not safe; but the value is small, the seismic effect is too large and uneconomical. The material type, cross section and vertical arrangement of the infill wall will affect the stiffness, and indirectly affect the period shortening factor.

Therefore, the reasonable value is necessary. The period shortening factor of solid clay brick and aerated concrete fill wall is in accordance with "Technical Specification for Concrete Structures of Tall Building ". All kinds of concrete hollow block infill wall for frame structure is 0.90 , frame shear structure is 0.95 , the shear wall structure is 1.00 , the value of the clay hollow block wall is $0.95 \sim 1.00$ [4].

\section{ANALYSIS AND ADJUSTMENT OF THE PARAMETERS IN CALCULATION RESULTS}

After the modeling and parameter setting, the calculation is completed. It analyses the calculation results of the text data and graphic data to ensure that the model and calculation results are correct, economical and reasonable. The parameters are shown in Fig. 1.

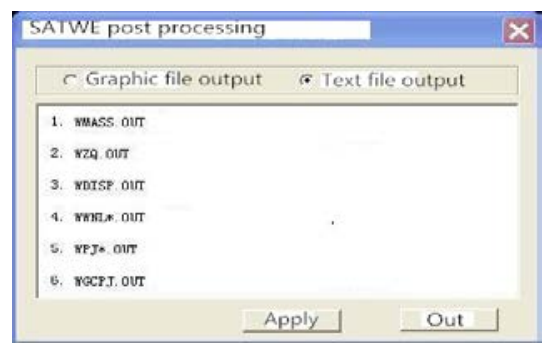

Fig. 1. Diagram of calculation parameter information 
Most students do not know what to do after the calculation is done. The software operation do not end here, the next work will analyze the calculation results and the reasonable solution to the problem. Generally it can not be a one-time calculation success, it needs to adjust and recalculate many times until all the indexes meet the requirements.

The control indexes of PKPM structure design are the text and graphic data of the calculation results. The graphic files can be used to look over the center location of the model quality and stiffness, the axial compression ratio of the column, the structural deformation diagram under various working conditions, the vibration of the whole space of the structure and the super reinforcement of the beam and column etc.

There are many contents in the text, the indexes are used to control the integrity of the structure, including axial compression ratio, period ratio, displacement ratio, stiffness ratio, the ratio of shear bearing capacity, stiffness to weight ratio, shear weight ratio. The students need to understand the meaning, the specific location in the text and the specification limit of each parameter etc. Some of the parameters in the text need to be looked at first, it is difficult to grasp and adjust, such as the period ratio, displacement ratio and axial compression ratio. Next, we analyze the control principle and adjustment method of these three parameters.

\section{A. Period Ratio}

Period ratio is not directly given in PKPM output file, so you need to calculate. For general single tower structure, the first and the second period is the translational period, and the third period is the first torsion period. The sum of the translational and torsion coefficient is equal to 1.00. If the translational coefficient is greater than 0.50 , it is translational mode. If the torsion coefficient is greater than 0.50 , it is torsion mode [5]. For some complex structures, it should be combined with the information of "The Whole Structure Spatial Vibration Map”.

If it is a multi-storey building, generally it does not consider the period ratio, only controls the displacement ratio and stiffness ratio. Most of the students' classroom exercises are multi-layer and regular buildings, but there are many problems in the calculation results. When the proportion of the torsion in the translational period is too large, the students cannot find the cause of problem and the period file, do not know how to modify it.

For example, in the first mode, the translational coefficient is 0.58 and the torsion coefficient is about 0.42 . The calculation result is not very reasonable. The first mode is the translational mode and the torsion proportion is significant, so the seismic performance is poor. The translational coefficient should be increased and the torsion coefficient should be reduced. The principle of adjustment should strengthen the stiffness of surrounding structural and weaken the middle wall, and make the center of mass and stiffness of the structure as close as possible. The peripheral component is uniform and symmetrical. The specification does not specify the value of the translational coefficient. It should be greater than 0.80 for the simple and regular multilayer structure.
When the period ratio doesn't meet the requirements, it is shown that the torsion stiffness of the structure is smaller than the lateral stiffness; the adjustment method is as follows.

1) Through the adjustment of the overall structure, it could strengthen the stiffness of walls, columns or beams of a peripheral structure (reduce the first torsion period).

2) It could weaken the stiffness of the middle wall and column of the structure (increase the first translational period).

3) It could reduce the stiffness of the lateral force resisting member if the direction of the spindle is on the short cycle, increase the lateral stiffness of the component increase the lateral stiffness of the component if it is on the contrary, and pay attention to the displacement ratio of each layer.

4) The layout of the surrounding structure should be uniform, symmetrical and continuous.

\section{B. Displacement Ratio}

The displacement ratio is mainly used to control the regularity of the structural plane. When the displacement ratio is too large, the regularity is poor. If the offset distance of the structure stiffness and quality center is large, it is easy to form the structural torsion effect, and form adverse effect on structure.

In the course of students practice, the most problem is the displacement ratio. When the displacement ratio is not satisfied, they do not know how to readjust and set the structure. First of all, the displacement ratio only takes account of the top displacement of the column and the wall, it is calculated under the assumption of rigid floor. It should need to note the unsatisfied floor, and clear adjustment of the location and objectives. The partial adjustment is more effective and economical than the overall adjustment. The adjustment method is as follows.

1) Strengthen the wall and column stiffness on the displacement point of the maximum, we can use the search function of the SATWE "The each floor numbering diagram of reinforcement member", determine the maximum displacement node fast, and then the node near the component stiffness are strengthened, the largest displacement node is usually easy to appear in the four corners of the structure [6].

2) Weaken the stiffness of the minimum displacement node.

3) The displacement ratio of $\mathrm{X}+, \mathrm{X}-, \mathrm{Y}+$ and $\mathrm{Y}$ - in four directions under accidental eccentricity is analyzed, which the $\mathrm{X}+$ is the right side of the structure, and the $\mathrm{Y}+$ is above the structure. You can see the specific direction of the limit, and then adjust the direction of the column or beam section size.

The accidental eccentricity is not taken into account when we calculate the displacement angle, because the result of accidental eccentricity is larger than the standard earthquake. However, in the process of teaching, most students calculate the displacement ratio and displacement angle are taken into account the accidental eccentricity, it will lead to the larger value of the member section, and the economy is poor. 


\section{Axial Compression Ratio}

The axial compression ratio can be seen in the output of the graphic file. When the axial compression ratio exceeds the limit, the ductility of the structure can not be guaranteed, it can increase the cross section column, wall or the concrete strength grade of the floor. The axial compression ratio is too small, which shows that the cross section of the column is larger and the economy is poor.

The standard does not specify the value of the axial compression ratio; the majority of students do not grasp the scope of the economy. The students can reserve the safety margin about $10 \% \sim 20 \%$ in the course of teaching. Such as the three earthquake resistant frame structure, the limit value of column axial compression ratio is 0.85 , this ratio between $0.6 \sim 0.8$ is appropriate.

\section{CONCLUSION}

The "Building Structure Computerization" is an important compulsory course for civil engineering major, which plays an important role in training students' professional knowledge and practical ability. In the course of teaching, there are too many design parameters. The students can't adjust the parameters and analyze the calculation results well, this ability is very weak, and it is difficult to master.

In view of the existing problems about the students, we have improved the teaching of the course, and the calculation parameters are divided into two categories according to the preprocessing and post-processing programs. The design parameters of the first kind are divided into four categories, and the number of modes and the period shortening factor are introduced mainly. This paper summarizes second kinds of parameters, and puts forward the use and adjustment of the period ratio, displacement ratio and axial compression ratio. After the improvement of the teaching method, the difficulty of the course study is reduced, and the students' learning interest is improved. It provides a good reference for the further reform of civil engineering practice teaching.

\section{ACKNOWLEDGMENT}

This work was supported by the education science "13th Five-year Plan" key topic of Fujian Province of China (The practice research of flipped classroom based on the microcourse, Project No. FJJKCGZ16-069).

\section{REFERENCES}

[1] Fan Xiaoping, "Several problems of PKPM software in building structure design,” Chongqing Architecture, vol. 52, pp. 28-30, Feb 2008.

[2] Ou Zelin, "Research on Several problems in the Design of Multistory building structures,” Science and Technology Information, vol. 23, pp. 312-314, June 2009.

[3] Lu Xiang-kun, and Wang Dong, "Problem that application PKPM structure design software should BE valued,” Sichuan Building Science, vol. 31, pp. 39-42, October 2005.

[4] Zhang Jing-shu, Jiang Li-na, and Feng Li-ping, "Research on Period Shortening Factor," Architecture Technology, vol. 41, pp. 49-52, January 2010.

[5] Deng Xiaqing, "The control and adjustment of six "Ratios" in the design of high rise structures-the comparison between the results of SATWE and the specification," Architectural Science, vol. 52, pp. 55-58, Feb 2009.

[6] Wei Lian, Wang Sen, and Wei Chengji, "Control of translation period and torsional period ratio of high-rise building,” Building Structure, vol. 44, pp. 1-4, March 2014. 